\title{
Wave Propagation Analysis in Composite Laminates Containing a Delamination Using a Three-Dimensional Spectral Element Method
}

\author{
Fucai Li, Haikuo Peng, Xuewei Sun, Jinfu Wang, and Guang Meng \\ State Key Laboratory of Mechanical System and Vibration, Shanghai Jiao Tong University, \\ Shanghai 200240, China \\ Correspondence should be addressed to Fucai Li, fcli@sjtu.edu.cn \\ Received 30 December 2011; Revised 13 February 2012; Accepted 23 February 2012 \\ Academic Editor: Zhongqing Su
}

Copyright (C) 2012 Fucai Li et al. This is an open access article distributed under the Creative Commons Attribution License, which permits unrestricted use, distribution, and reproduction in any medium, provided the original work is properly cited.

\begin{abstract}
A three-dimensional spectral element method (SEM) was developed for analysis of Lamb wave propagation in composite laminates containing a delamination. SEM is more efficient in simulating wave propagation in structures than conventional finite element method (FEM) because of its unique diagonal form of the mass matrix. Three types of composite laminates, namely, unidirectional-ply laminates, cross-ply laminates, and angle-ply laminates are modeled using three-dimensional spectral finite elements. Wave propagation characteristics in intact composite laminates are investigated, and the effectiveness of the method is validated by comparison of the simulation results with analytical solutions based on transfer matrix method. Different Lamb wave mode interactions with delamination are evaluated, and it is demonstrated that symmetric Lamb wave mode may be insensitive to delamination at certain interfaces of laminates while the antisymmetric mode is more suited for identification of delamination in composite structures.
\end{abstract}

\section{Introduction}

Owing to its superior mechanical properties and light weight, composite materials are finding more and more applications especially in aerospace industries [1]. However, composite structures still run a high risk of suffering from damage due to abrupt impact or growth of fatigue defects, which can result in catastrophic failure during their service life. It is, therefore, essential to develop techniques to inspect integrity and improve safety, reliability, and operational life of structures [2-7].

Traditionally, nondestructive evaluation (NDE) techniques, such as C-scan and radiographic inspection, are used to evaluate the integrity and degradation of structures on a periodic basis. Now online structural health monitoring (SHM) techniques, for example, 
vibration-based and Lamb-wave-based techniques, are being developed to provide early warning and assure the performance of structures. Among them, Lamb-wave-based techniques for damage detection have received a considerable attention in the past decades due to its ability of long-distance propagation and sensitivity to a variety of defects.

For Lamb waves-based damage detection techniques, understanding wave propagation characteristics in structures is essential for their successful application. Therefore, a number of numerical methods have been applied to analyze propagation of elastic waves, such as finite difference method (FDM) [8,9], finite element method (FEM) [10-12], boundary element method (BEM) $[13,14]$, finite strip elements (FSE) $[15,16]$, mass-spring lattice model (MSLM) [17-21], and local interaction simulation approach (LISA) [22, 23].

In the literature, two kinds of spectral element method (SEM) were applied to wave propagation modeling, namely, fast-Fourier-transform- (FFT-) based SEM and the orthogonal polynomials-based SEM [24-26]. In the FFT-based SEM [24], a single element is sufficient to model wave propagation in large uniform structures, which is suited for simple 1D and 2D problems [27]. On the other hand, the orthogonal-polynomials- (e.g., Legendre and Cheybysev polynomials) based SEM [25] is much more suitable for analyzing wave propagation in structures with complex geometry. The formulation of the spectral finite element (SFE) is similar to FE when assembly of element matrices and solution of equations are considered. Hence, the SEM can be used to handle the wave propagation in structures with complex geometry, and various types of defects can be modeled. The difference between the SEM and FEM comes in that orthogonal polynomials are used as approximation functions in SEM and, therefore, calculation can be more efficient because of the diagonal mass matrix. This method has been successfully applied to many fields, such as fluids, seismology and acoustics $[28,29]$. More recently, the SEM was used to simulate wave propagation in structures for damage detection, for example, wave propagation in 1D structures, such as rod and beam $[30,31]$. Numerical simulation results of the elastic wave propagation in a composite plate were presented by Kudela et al. [32]. A 2D spectral membrane finite element-based model, developed by Zak et al. [33], was used to analyze wave propagation in a cracked isotropic panel. Wave propagation in 2D plate structures using a 3D SEM for damage detection was also discussed by Peng et al. [34].

A wave propagation analysis in composite structures using 3D SEM for the purpose of damage detection has not been widely reported in the literature so far. Although characteristics of Lamb wave propagation in composite laminates and the damage evaluation using numerical simulation have been rigorously explored for a couple of years, in most of the related work, structures were simplified by either one- or two-dimensional models, resulting in approximate results especially for laminates of complicated layup or with damage. The SEM combines accuracy with flexibility in describing problems with complex geometries, which is highly desirable for modeling of elastic wave propagation. In this paper, multilayered composite laminates are modeled using the Legendre polynomials-based spectral finite element, elastic wave propagation characteristics are analyzed, and wave interaction with delamination is discussed.

\section{Wave Propagation in Composite Plate}

Composite laminates are commonly fabricated by stacking unidirectional lamina with a certain layup configuration. After a composite is properly cured, a multilayered structure is formed with all the layers bonded together. For analysis of wave propagation, each lamina 


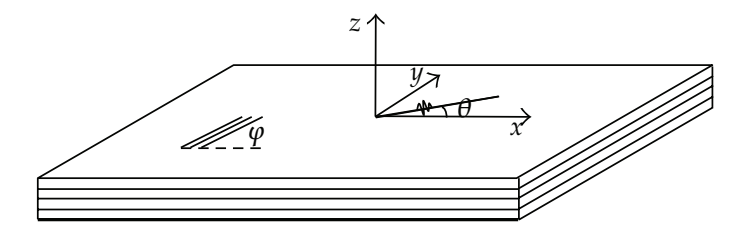

Figure 1: Sketch of a multilayered structure and the coordinate system.

can be regarded as a quasihomogeneous orthotropic or transversely isotropic material with the main principal axis parallel to the fibres. Therefore, in numerical calculations, a composite laminate is modeled as a multilayered medium with different elastic and anisotropic material properties [35].

In order to form a numerical model of composite structure, a Cartesian coordinate system is firstly defined by the $z$-axis normal to the central plane of a composite laminate spanned by the $x$-axis and the $y$-axis for modeling of the wave propagation, as shown in Figure 1.

For each layer of the composite laminate, the stress-strain relations for arbitrary direction have the following form [36]:

$$
\sigma=D \varepsilon,
$$

where $\sigma$ is stress vector, $\varepsilon$ is strain vector, and $D$ is the flexibility matrix. In order to study wave propagation, the elastic constants of all the layers must be expressed in the global coordinate system. For those layers, the principal material coordinate system does not coincide with the global coordinate system; this can be achieved by using a transformation matrix method.

In a homogeneous media, the elastic wave propagation is described by the governing equation [37]:

$$
\rho \partial_{t}^{2} \mathbf{u}=\nabla \cdot \sigma+\mathbf{f}
$$

where $\mathbf{u}$ is the displacement vector. $\rho$ is mass density and $\mathbf{f}$ is external force vector.

\section{Formulation of 3D Spectral Element Method}

For the Legendre polynomials-based 3D spectral finite element, it requires that the domain $\Omega$ in three dimensions is decomposed into a number of nonoverlapping hexahedrons, $\Omega_{e}$, as in the conventional FE method. In SEM, the equations of wave propagation is [37]

$$
\int_{\Omega} \rho \mathbf{w} \cdot \partial_{t}^{2} \mathbf{u} d \Omega+\int_{\Omega} \nabla \mathbf{w}: \mathbf{C}: \nabla \mathbf{u} d \Omega=\int_{\Omega} \mathbf{w} \cdot \mathbf{f} d \Omega
$$

where the $\Omega$ denotes the physical region of interest and $\mathbf{w}$ is an arbitrary test vector.

In SEM, the nodes are defined into two steps: (1) each element in its physical domain is mapped to a reference domain $\Lambda=[-1,1]^{3}$ using an invertible local mapping $f$; a set of basis functions consisting of Legendre polynomials of degree $N$ are introduced; (2) a set of 


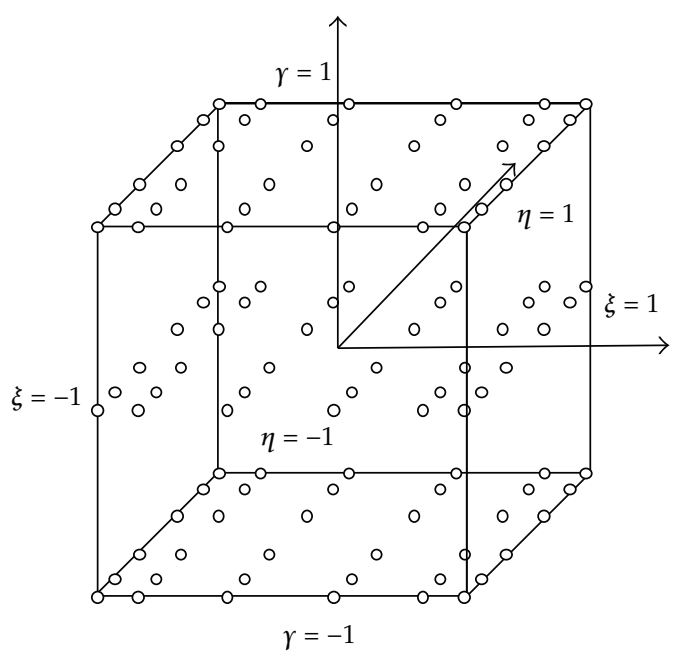

Figure 2: A 108-node spectra element in the local coordinate.

nodes are defined as $\xi_{i} \in[-1,1], i \in 1, \ldots, N+1$. These Gauss-Lobatto-Legendre (GLL) points are the $(N+1)$ roots of [29]

$$
\left(1-\xi^{2}\right) P_{N}^{\prime}(\xi)=0
$$

where $P_{N}^{\prime}(\xi)$ is the derivative of the Legendre polynomial of degree $N$. The $\xi_{i}$ are different from the classical FE method in which the nodes are uniformly spaced. As an example, a 108-node spectral element in the local coordinate is shown in Figure 2.

The Lagrange interpolation function, $u_{N}^{e}$, supported by the GLL points can be expressed as

$$
\begin{aligned}
u_{N}^{e}(\xi, \eta, \gamma) & =\sum_{m=1}^{n_{1}} \sum_{n=1}^{n_{2}} \sum_{r=1}^{n_{3}} u_{N}^{e}\left(\xi_{m}, \eta_{n}, \gamma_{r}\right) h_{m}(\xi) h_{n}(\eta) h_{r}(\gamma) \\
& =\sum_{m=1}^{n_{1}} \sum_{n=1}^{n_{2}} \sum_{r=1}^{n_{3}} u_{N}^{e}\left(\xi_{m}, \eta_{n}, \gamma_{r}\right) \Psi_{m n r}
\end{aligned}
$$

where $\Psi_{m n r}$ is defined as the orthogonal shape functions in 3-D $h_{m}(\xi)$ denotes the $m$ th 1D Lagrange interpolation at the $(N+1)$ GLL points defined above. The property of $h_{m}(\xi)$ is

$$
h_{m}\left(\xi_{n}\right)=\delta_{m n}
$$

where $\delta_{m n}$ denotes the Kronecker delta and $n_{i}, i=1,2,3$, are the numbers of GLL points in each direction in the local coordinate. 
Therefore, the element matrices, $\mathbf{M}^{e}$ (mass matrix), $\mathbf{K}^{e}$ (stiffness matrix), and $\mathbf{F}^{e}$ (force vectors), are calculated numerically on the reference coordinate:

$$
\begin{aligned}
\mathbf{M}^{e} & =\rho \int_{\Omega_{e}}\left(\Psi^{e}(x, y, z)\right)^{T}\left(\Psi^{e}(x, y, z)\right) d \Omega_{e} \\
& =\rho \int_{-1}^{+1} \int_{-1}^{+1} \int_{-1}^{+1}\left(\Psi^{e}(\xi, \eta, \gamma)\right)^{T}\left(\Psi^{e}(\xi, \eta, \gamma)\right) \operatorname{det}\left[\mathbf{J}_{e}\right] d \xi d \eta d \gamma \\
& =\rho \sum_{i=1}^{n_{1}} \omega_{i} \sum_{j=1}^{n_{2}} \omega_{j} \sum_{k=1}^{n_{3}} \omega_{k}\left[\Psi^{e}\left(\xi_{i}, \eta_{j}, \gamma_{k}\right)\right]^{T}\left[\Psi^{e}\left(\xi_{i}, \eta_{j}, \gamma_{k}\right)\right] \operatorname{det}\left[\mathbf{J}_{e}\right] \\
\mathbf{K}^{e} & =\int_{\Omega_{e}}\left(\mathbf{B}^{e}(x, y, z)\right)^{T} \mathbf{D}^{e}\left(\mathbf{B}^{e}(x, y, z)\right) d \Omega_{e} \\
& =\int_{-1}^{+1} \int_{-1}^{+1} \int_{-1}^{+1}\left(\mathbf{B}^{e}(\xi, \eta, \gamma)\right)^{T} \mathbf{D}^{e}\left(\mathbf{B}^{e}(\xi, \eta, \gamma)\right) \operatorname{det}\left[\mathbf{J}_{e}\right] d \xi d \eta d \gamma \\
& =\sum_{i=1}^{n_{1}} \omega_{i} \sum_{j=1}^{n_{2}} \omega_{j} \sum_{k=1}^{n_{3}} \omega_{k}\left[\mathbf{B}^{e}\left(\xi_{i}, \eta_{j}, \gamma_{k}\right)\right]^{T} \mathbf{D}^{e}\left[\mathbf{B}^{e}\left(\xi_{i}, \eta_{j}, \gamma_{k}\right)\right] \operatorname{det}\left[\mathbf{J}_{e}\right] \\
\mathbf{F}^{e} & =\int_{\Omega_{e}}\left(\Psi^{e}(x, y, z)\right)^{T} \mathbf{P} d \Omega_{e} \\
& =\int_{-1}^{+1} \int_{-1}^{+1} \int_{-1}^{+1}\left(\Psi^{e}(\xi, \eta, \gamma)\right) \mathbf{P}(\xi, \eta, \gamma) \operatorname{det}\left[\mathbf{J}_{e}\right] d \xi d \eta d \gamma \\
& =\sum_{i=1}^{n_{1}} \omega_{i} \sum_{j=1}^{n_{2}} \omega_{j} \sum_{k=1}^{n_{3}} \omega_{k}\left[\Psi^{e}\left(\xi_{i}, \eta_{j}, \gamma_{k}\right)\right]^{T} \mathbf{P}\left(\xi_{i}, \eta_{j}, \gamma_{k}\right) \operatorname{det}\left[\mathbf{J}_{e}\right]
\end{aligned}
$$

where $\rho$ is the mass density, $\mathbf{D}^{e}$ is termed material stiffness matrix, and $\mathbf{P}$ is a distributed load. $\Psi^{\mathrm{e}}$ are the shape functions based on the Legendre polynomials. The matrix $\mathbf{B}^{e}$ is the strain-displacement matrix calculated by

$$
\mathbf{B}^{e}=\mathbf{L} \Psi^{e}(x, y, z),
$$

where $\mathbf{L}$ denotes a differential operator matrix:

$$
\mathbf{L}=\left[\begin{array}{cccccc}
\partial_{x} & 0 & 0 & \partial_{y} & 0 & \partial_{z} \\
0 & \partial_{y} & 0 & \partial_{x} & \partial_{z} & 0 \\
0 & 0 & \partial_{z} & 0 & \partial_{y} & \partial_{x}
\end{array}\right]^{T}
$$


$\mathbf{J}_{e}$ is the Jacobian matrix associated with the mapping $f$ from the physical domain $\Omega_{\mathrm{e}}$ to the reference domain $\Lambda$ :

$$
\mathbf{J}_{e}=\left[\begin{array}{lll}
\partial_{\xi} x & \partial_{\xi} y & \partial_{\xi} z \\
\partial_{\eta} x & \partial_{\eta} y & \partial_{\eta} z \\
\partial_{\gamma} x & \partial_{\gamma} y & \partial_{\gamma} z
\end{array}\right]
$$

The weights $\omega_{i}$ are defined by

$$
\omega_{i}=\frac{2}{n(n-1)\left[P_{n-1}\left(\xi_{i}\right)\right]^{2}}, \quad i \in 1, \ldots, n, n=N+1
$$

Therefore, wave equation (3.1) can be rewritten into matrix form and wave propagation modeling is transformed to an ordinary differential equation in time. Let $\mathbf{U}$ denotes the global vector of unknown displacement in the medium. Then the ordinary differential equation can be written as

$$
\mathbf{M U ̈}+\mathbf{K U}=\mathbf{F},
$$

where $\mathbf{M}$ denotes the global mass matrix, $\mathbf{K}$ is the global stiffness matrix, and $\mathbf{F}$ is the vector of time-dependent excitation force.

In this study, the differential equation (3.10) is solved using a central difference time integration scheme. Initial conditions of displacement and velocity are $\mathbf{U}=0$ and $\mathbf{U}=0$ at the time $t=0$, and the central difference time integration scheme is implemented as

$$
\frac{1}{\Delta t^{2}} \mathbf{M U}_{t+\Delta t}=\mathbf{F}_{t}-\left(\mathbf{K}-\frac{2}{\Delta t^{2}} \mathbf{M}\right) \mathbf{U}_{t}-\frac{1}{\Delta t^{2}} \mathbf{M U}_{t-\Delta t}
$$

where the symbol $t$ denotes time and $\Delta t$ denotes the time step of integration. In the central difference time integration scheme, the stable condition is $\Delta t \leq \Delta t_{c r}=L / c$, where $L$ is the minimum distance between two adjacent nodes and $c$ is the wave speed in elastic medium.

In comparison with FE, less computation effort is required for SFE because of the choice of Lagrange interpolation supported on the GLL points in conjunction with the GLL integration rule. The efficiency of SEM was demonstrated using two 3D models based on SFEs and FEs with the same degrees of freedom, and a reduction of about $65 \%$ in CPU time for calculation is used in comparison with the FEM.

\section{Numerical Calculation}

Lamb wave propagation in 8-ply T300/F593 composite laminates is analyzed in this study. The material properties of unidirectional lamina are listed in Table 1. Three types of composite laminates, namely, unidirectional $\left[0_{8}\right]$, symmetric cross-ply $\left[0_{2} / 90_{2}\right]_{s}$, and quasi-isotropic $[+45 / 45 / 0 / 90]_{s}$ laminates are investigated. All the laminates have the same geometric configurations of $500 \mathrm{~mm} \times 500 \mathrm{~mm} \times 1.72 \mathrm{~mm}$, as shown in Figure 3. The composite plate 


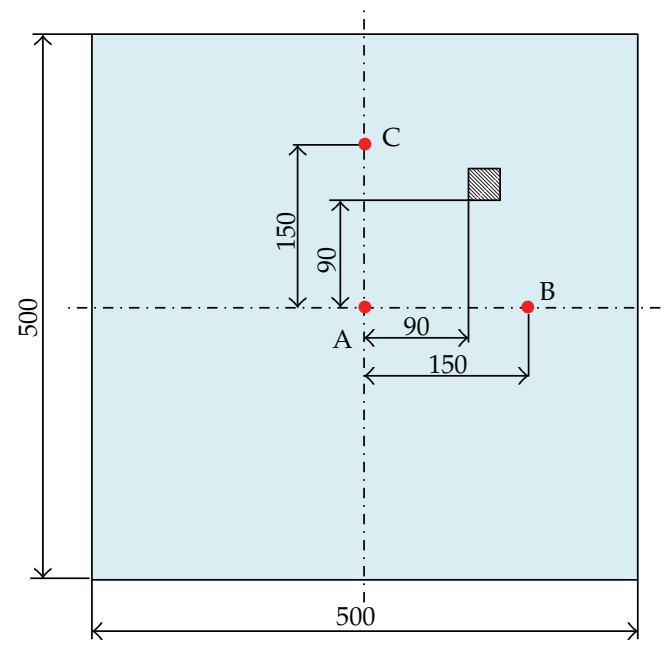

Figure 3: Scheme of composite laminate containing a delamination.

Table 1: Material properties of composite lamina.

\begin{tabular}{lccccccccc}
\hline$E_{1}(\mathrm{GPa})$ & $E_{2}(\mathrm{GPa})$ & $E_{3}(\mathrm{GPa})$ & $G_{12}(\mathrm{GPa})$ & $G_{13}(\mathrm{GPa})$ & $G_{23}(\mathrm{GPa})$ & $v_{12}$ & $v_{13}$ & $v_{23}$ & $\rho\left(10^{3} \mathrm{~kg} / \mathrm{m}^{3}\right)$ \\
\hline 128.1 & 8.2 & 8.2 & 4.7 & 4.7 & 3.44 & 0.27 & 0.27 & 0.2 & 1.57 \\
\hline
\end{tabular}

was meshed using the three-dimensional spectral finite elements with 108-node (shown in Figure 2).

In the literature, piezoelectric (PZT) wafer is one of the frequently applied transducers to excite and capture Lamb waves propagating in structures for damage detection. The PZT transducers can be modeled by adding force to the incident points or using electromechanical coupling for elastic wave modeling in structures [38, 39]. In the present study, in order to obtain relatively simple Lamb wave mode, two forces perpendicular to the plane of the plate are applied at point $\mathrm{A}$ in Figure 3 on the upper and the lower surfaces of the plate, respectively. When the two forces are in phase, antisymmetric modes are activated, and when the two forces are out of phase, symmetric modes are excited. The excitation force is a 5-cycle sinusoidal signal modulated by Hanning window with a center frequency of $100 \mathrm{kHz}$ and absolute maximum magnitude is $1 N$, as shown in Figure 4 . The displacement responses at point $B$ and $C$ on the upper surface of the composite laminate are used to investigate wave propagation characteristics.

\subsection{Wave Propagation in a Multilayered Composite Plate}

As aforementioned, three types of the composite laminates are analyzed in this study. Firstly, Lamb wave propagation in the unidirectional composite laminate $[0]_{8}$ is investigated. The plate is meshed to $50 \times 50 \times 1$ spectral elements. Under the two excitation of in-phase forces, the fundamental antisymmetric mode $\mathrm{A}_{0}$ is excited. According to the simulation results, the displacement component in the $z$-direction is dominant. Therefore, only the displacement component in the $z$-direction is analyzed here. Responses of the laminate at $0.097 \mathrm{~ms}$ are 


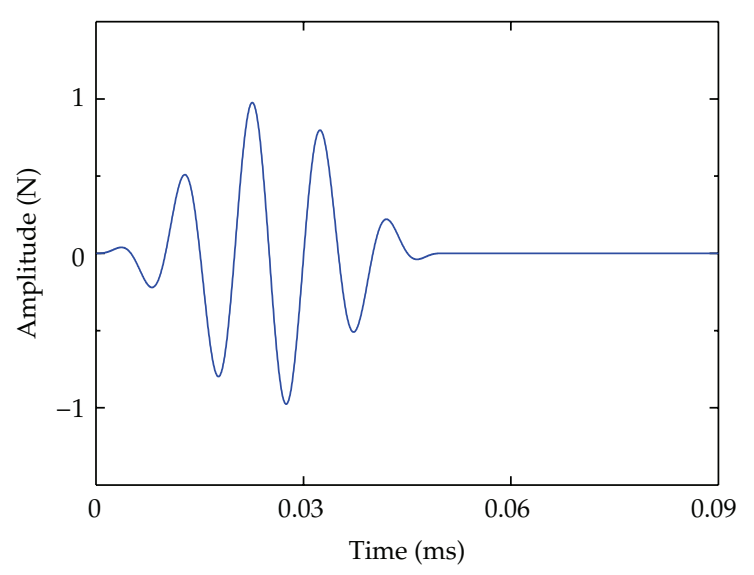

Figure 4: Waveform of the excitation force in the time domain.

plotted in Figure 5(a). It can be observed that the wave front of the $A_{0}$ mode has ellipselike shape, and the group velocity in the fibre direction is greater than that in the direction perpendicular to the fibre, indicating that group velocity of this type of wave mode in the composite depends on the orientation of wave propagation. Generally, group velocity in composite laminate is a function of the direction of wave propagation and direction of the fibres. The group velocity in composite laminates can be calculated analytically using the transfer matrix method (In the appendix). For such an 8-ply unidirectional laminate, the analytical group velocity is plotted in a polar coordinate, as shown in Figure 5(b). It presents similar feature as Figure 5(a). Group velocities in the direction along the fibers ( $x$-axis) and perpendicular to the fibres ( $y$-axis) are further calculated from displacement responses of SEM simulations at the point $B$ and $C$, as shown in Figure 6. Based on the peak of the received responses, time of flight (ToF) from point $\mathrm{A}$ to $\mathrm{B}$ can be defined. The calculated group velocity $c_{g}$ of the $\mathrm{A}_{0}$ mode of Lamb waves propagating in the $x$ direction is $1794 \mathrm{~m} / \mathrm{s}$, which is $2.6 \%$ smaller than the analytical value. In a similar way, the group velocity of the $\mathrm{A}_{0}$ mode in the $y$-direction is $1319 \mathrm{~m} / \mathrm{s}$ and the one calculated analytically is $1245 \mathrm{~m} / \mathrm{s}$, which gives a relative error of $5.9 \%$. It can be concluded that the simulation results of SEM model, the proposed model, are in good agreement with the analytical results thus validating the effectiveness of the model.

Under the two excitations of out-of-phase forces, the fundamental symmetric wave modes, the $\mathrm{S}_{0}$ mode and the $\mathrm{SH}_{0}$ mode, are excited. According to the simulation results, the displacement components in the $x$-direction and in the $y$-direction are dominated. Hence, only those two displacement components are plotted, as shown in Figures 7(a) and 7(b). It can be seen that $\mathrm{S}_{0}$ mode and the $\mathrm{SH}_{0}$ mode are excited simultaneously, which is because the $\mathrm{S}_{0}$ mode and the $\mathrm{SH}_{0}$ mode are coupled in multilayered composite laminate. Analytical group velocities of the $\mathrm{S}_{0}$ mode and the $\mathrm{SH}_{0}$ mode are also calculated, as shown in Figure 7(c), and the simulation results of SEM modeling are in good agreement of the analytical results.

In case of cross-ply laminates $\left[0_{2} / 90_{2}\right]_{s}$, the laminate was meshed to $50 \times 50 \times 4$ spectral finite elements. The symmetric mode and antisymmetric modes are excited using the above-mentioned method. In case of antisymmetricmode, the displacement component in the $z$-direction is shown in Figure 8. On the other hand, in case of symmetric mode the displacement in the $x$-direction and the $y$-direction are plotted in Figure 9. The complexity 


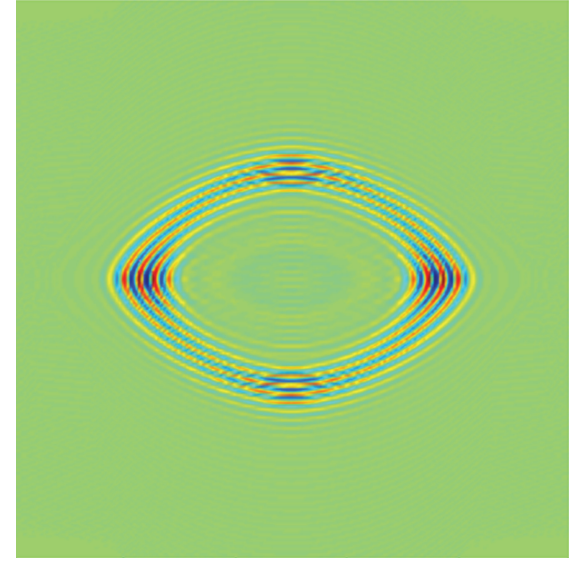

(a)

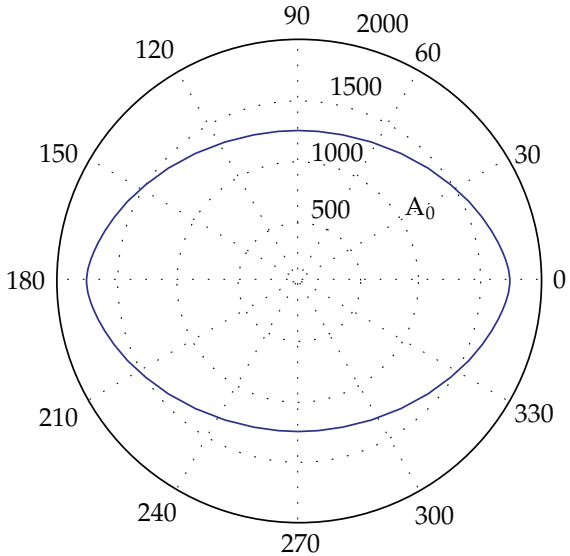

(b)

Figure 5: Displacement component in the $z$-direction at $0.097 \mathrm{~ms}$ under (a) the $\mathrm{A}_{0}$ mode and (b) theoretical group velocity.

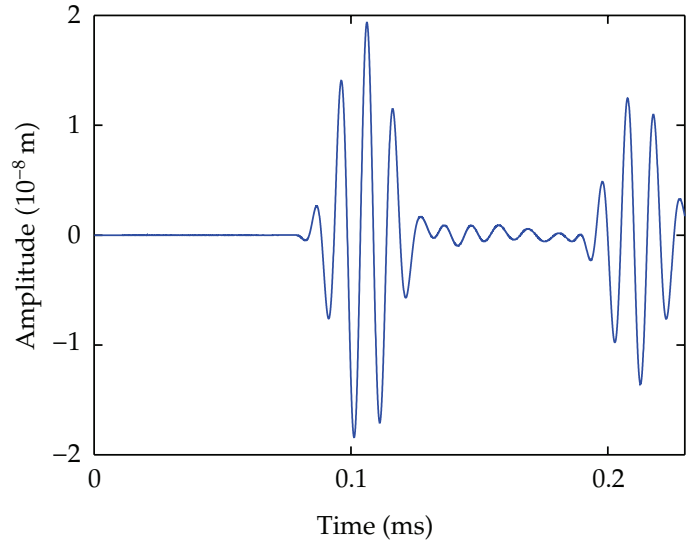

(a)

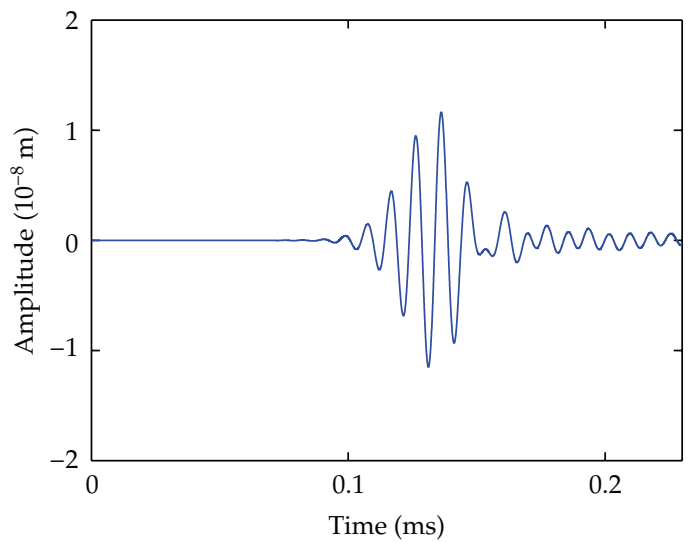

(b)

Figure 6: Displacement response in the $z$-direction at point B (a) and at point C (b).

of Lamb wave propagation in composite is also demonstrated, and the effectiveness of the proposed model is validated by comparison of simulation results and the analytical results.

In case of angle-ply laminates $[-45 / 45 / 0 / 90]_{s}$, the composite laminate is meshed by using $50 \times 50 \times 8$ spectral finite elements. The displacement components in the $z$-direction at $0.086 \mathrm{~ms}$ are plotted in Figure 10(a), while displacement components in the $x$-direction and in the $y$-direction at $0.069 \mathrm{~ms}$ are plotted in Figures 11(a) and 11(b). The group velocities of analytical solutions for those Lamb modes are also plotted in a polar coordinate for comparison, as shown in Figures 10(b) and 11(c). A good agreement found in both symmetric and antisymmetricmodes. It can be observed that the angular dependence of Lamb nodes in the laminates $[-45 / 45 / 0 / 90]_{S}$ becomes weaker because of its quasi-isotropic layup. Under the $\mathrm{S}_{0}$ and the $\mathrm{SH}_{0}$ modes, the group velocities are approximately independent of direction of wave propagation, but it still can be discerned that the $A_{0}$ mode has the maximum in the $45^{\circ}$ 


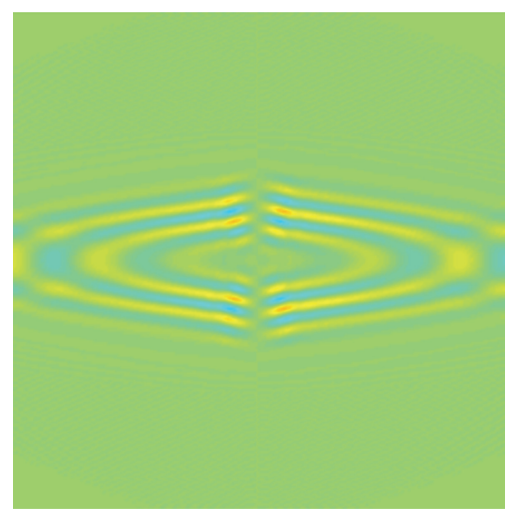

(a)

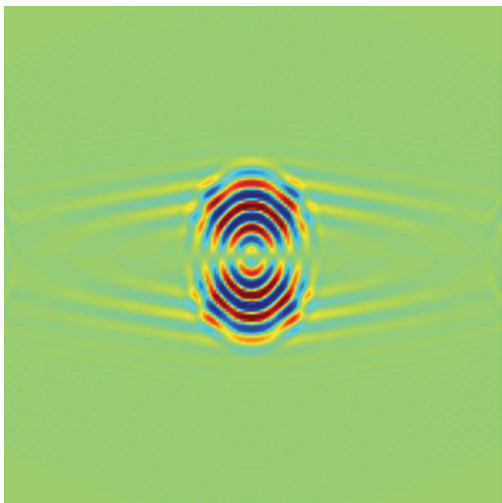

(b)

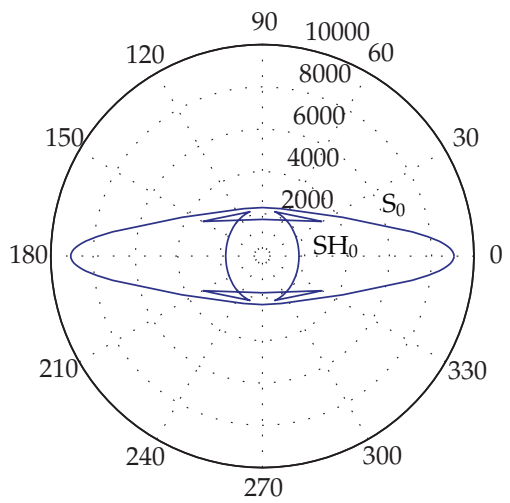

(c)

Figure 7: Displacement components in (a) the $x$-direction and (b) the $y$-direction at 0.039 ms under the $S_{0}$ and the $\mathrm{SH}_{0}$ modes and (c) theoretical group velocity.

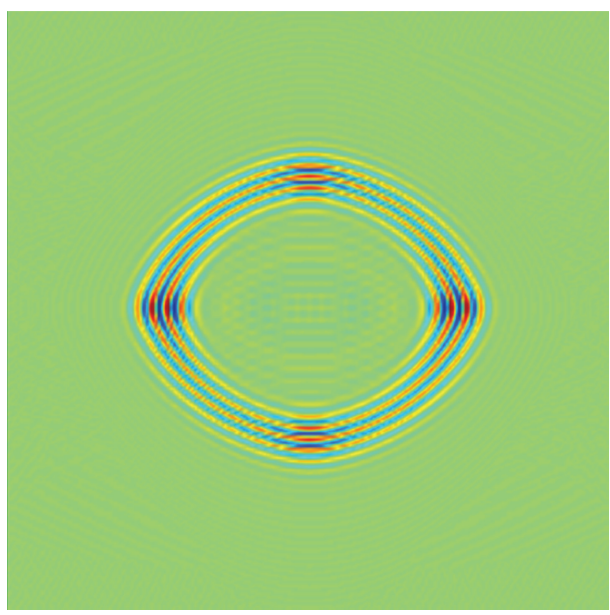

(a)

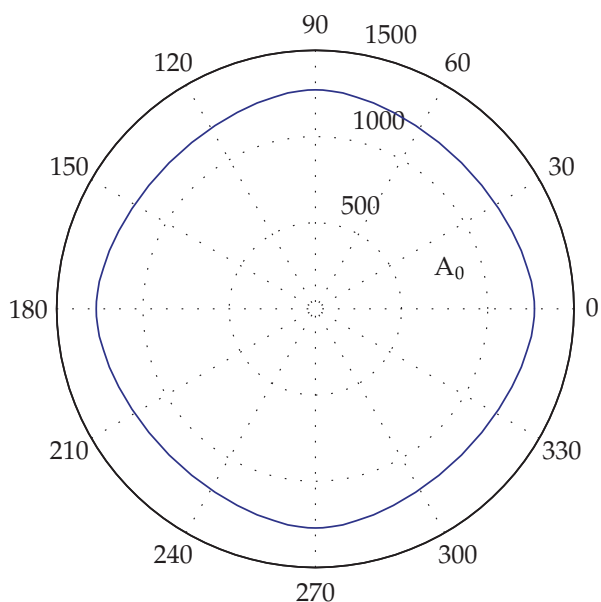

(b)

Figure 8: Displacement component in the $z$-direction at $0.097 \mathrm{~ms}$ under (a) the $\mathrm{A}_{0}$ mode and (b) analytical group velocities. 


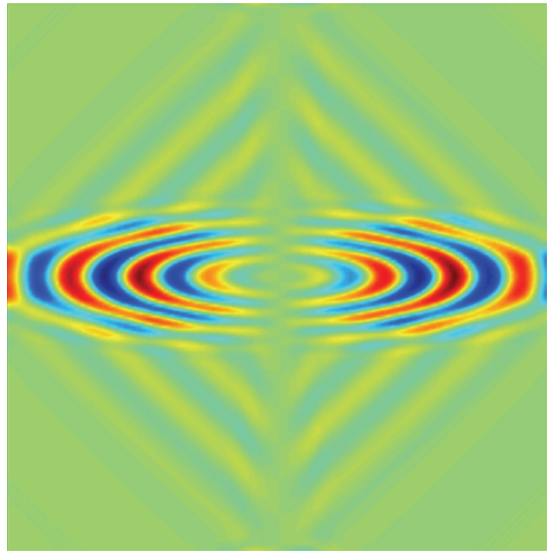

(a)

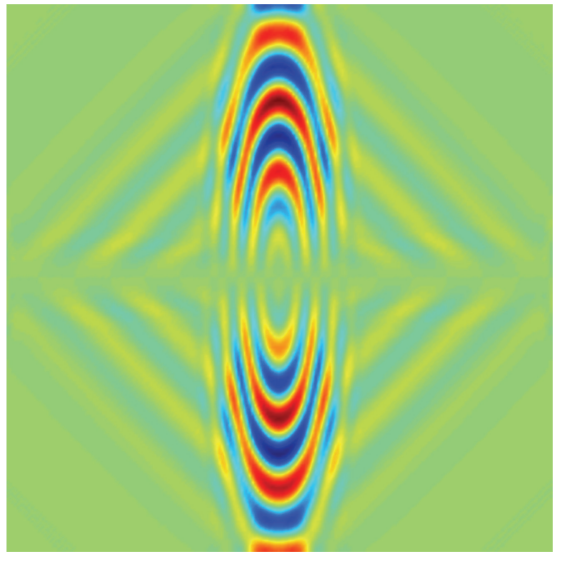

(b)

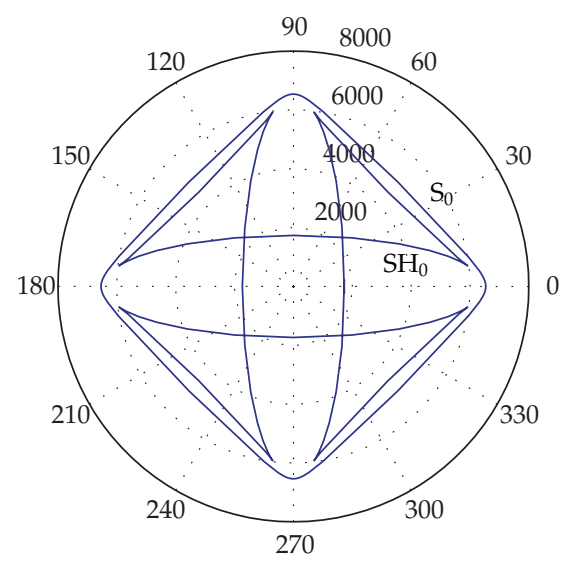

(c)

Figure 9: Displacement components in (a) the $x$-direction and in (b) the $y$-direction at $0.097 \mathrm{~ms}$ under the $\mathrm{S}_{0}$ and the $\mathrm{SH}_{0}$ modes and (c) theoretical group velocity.

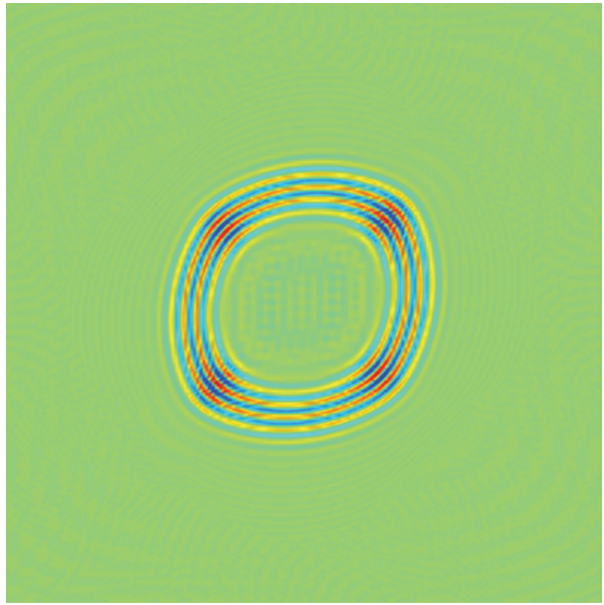

(a)

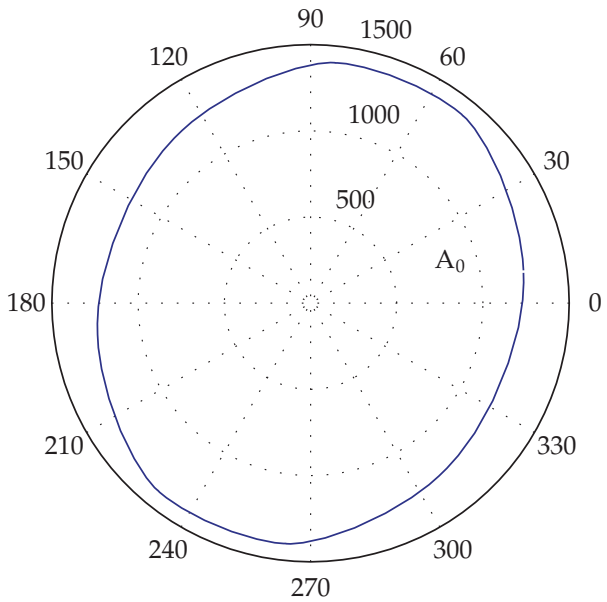

(b)

Figure 10: Displacement component in (a) the $z$-direction at $0.086 \mathrm{~ms}$ under the $A_{0}$ mode and (b) analytical group velocities. 
$\left(\right.$ or $225^{\circ}$ ) directions because outer lamina are orientated in these directions, which dominates the bending properties related to $\mathrm{A}_{0}$ mode.

In case of angle-ply laminate, the wavelength of the $\mathrm{A}_{0}$ mode is shorter than that of the $\mathrm{S}_{0}$ and the $\mathrm{SH}_{0}$ modes in composite laminate from the simulation results and analytical results. It can be expected that the $\mathrm{A}_{0}$ mode is possibly more sensitive to small damage than the $\mathrm{S}_{0}$ mode and the $\mathrm{SH}_{0}$ mode because of its short wavelength.

It is demonstrated that characteristics of the wave propagation in multilayered composite is complex due to the nature of anisotropic of the material constants and the multilayered configurations, which leads to the group velocity of Lamb waves depending on the laminate layup and the direction of wave propagation. Good agreement between the simulation results based on SEM and the analytical results demonstrates that the proposed model provides an effective tool to investigate the wave propagation in composite structures.

\subsection{Wave Propagation in a Composite Plate Containing Delamination}

The typical damage forms in composite laminate are transverse microcracking, fiber-breakage, and delamination. Typically, the transverse microcracking through the thickness of the ply occurs as the first-ply failure, and then delamination damage follows. The fiber breakage usually happens at the last stage of the failure. However, a catastrophic failure can occur only with the microcracking and delamination damage without the fiber breakage. Delamination is known to happen because of excessive interlamina normal and shear stress at the ply boundaries, which not only causes reduction in stiffness, but also affects the strength and integrity of the structure, leading to failure.

The wave propagation in composite laminates containing a delamination is investigated in this study. The delamination in the laminates is modeled using nodes separation method. Laminate without delamination is initially meshed. At the interface between the adjacent elements where the delamination occurs, nodes that are affected by the delamination are separated, as shown in Figure 12.

Lamb wave modes interaction with delamination is analyzed in quasi-isotropic laminates $[45 /-45 / 0 / 90]_{s}$ using the proposed 3D SEM model. The size of the delamination is $30 \mathrm{~mm} \times 30 \mathrm{~mm}$ in a square shape, as shown in Figure 3 . The wave interaction of the symmetric mode and antisymmetricmode with delamination is investigated.

The effects of the delamination at different interfaces in the composite laminate are addressed. Under the symmetric mode, the displacement responses at the point B in the $x$ and the $y$-directions are plotted in Figure 13. Responses of the intact composite laminate are also provided for comparison. It is evident that the scattered waves from the delamination are not so obvious in comparison with the intact laminate, on the captured responses at the point $B$, when the delamination is located in different interfaces. The displacement responses at $0.069 \mathrm{~ms}$ in the $x$ - and the $y$-directions of the composite laminates are plotted in Figure 14, when there is a delamination at the interfaces between 3-4 layers. However, when the delamination is at the interface between $4-5$ layers, responses of the point $B$ are same as those from intact composite laminate, indicating that the symmetric mode is insensitive to the delamination in the symmetric plane of the plate, attributed to the reason that the layup of the composite laminate is symmetric about the central plane between 4-5 layers. Therefore, the symmetric wave mode related to extensional wave mode can travel through the delamination without any scattering from it.

Under the antisymmetricmode, the displacements at the point B in the $z$-direction are plotted in Figure 15. It can be seen that the antisymmetricmode is more sensitive to 


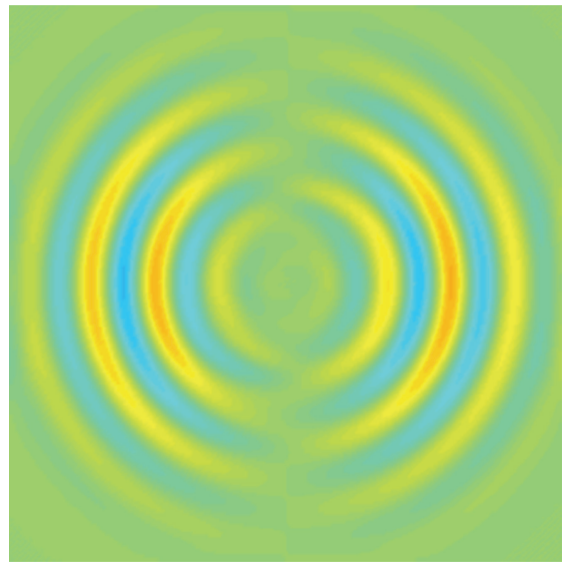

(a)

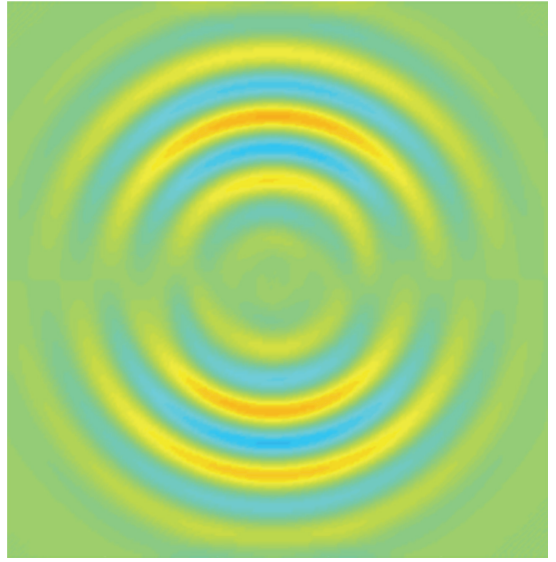

(b)

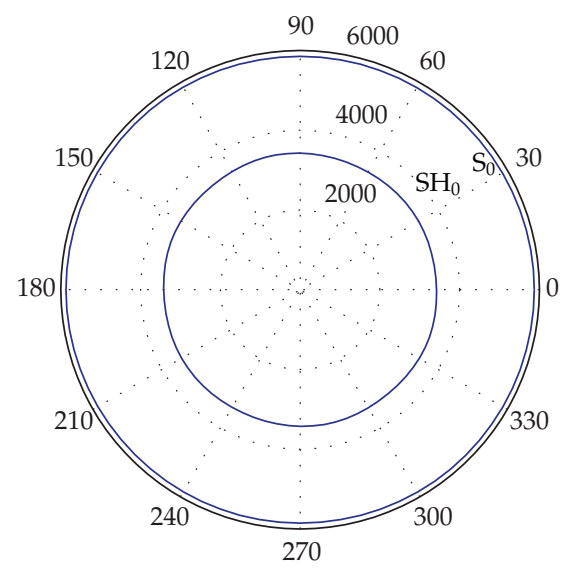

(c)

Figure 11: Displacement components in (a) the $x$-direction and in (b) the $y$-direction at 0.069 ms under the $\mathrm{S}_{0}$ and the $\mathrm{SH}_{0}$ modes and (c) theoretical group velocity.

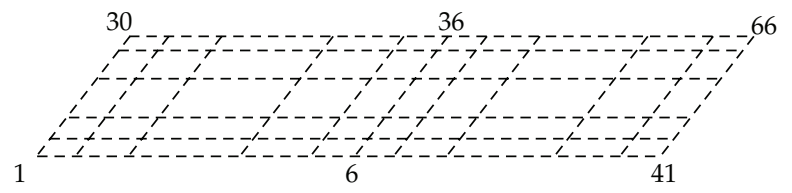

(a)

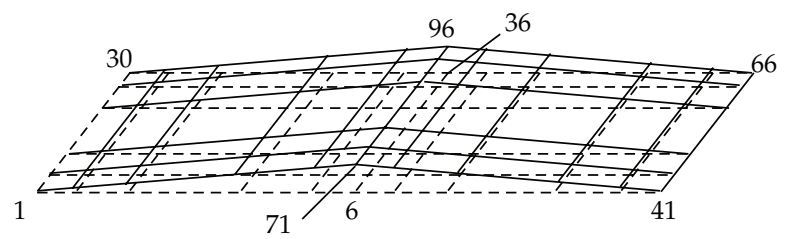

(b)

Figure 12: Modeling of delamination in a laminate (a) mesh without delamination and (b) mesh with a delamination. 


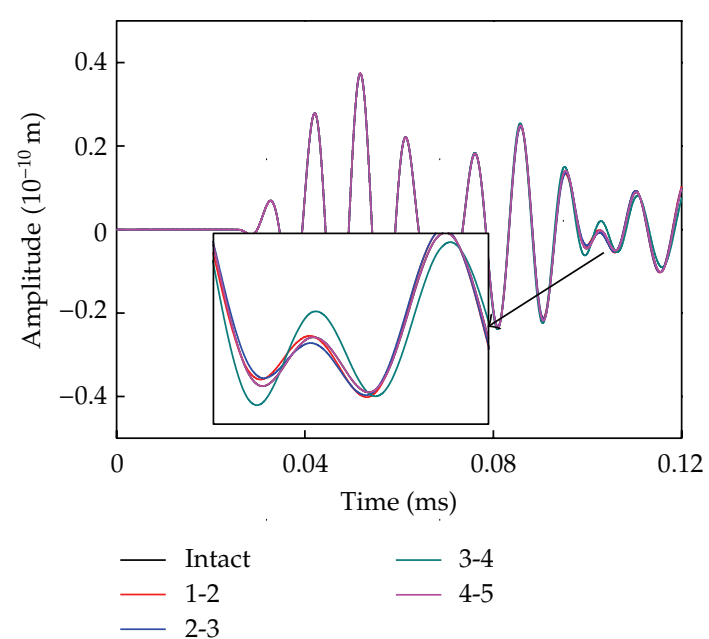

(a)

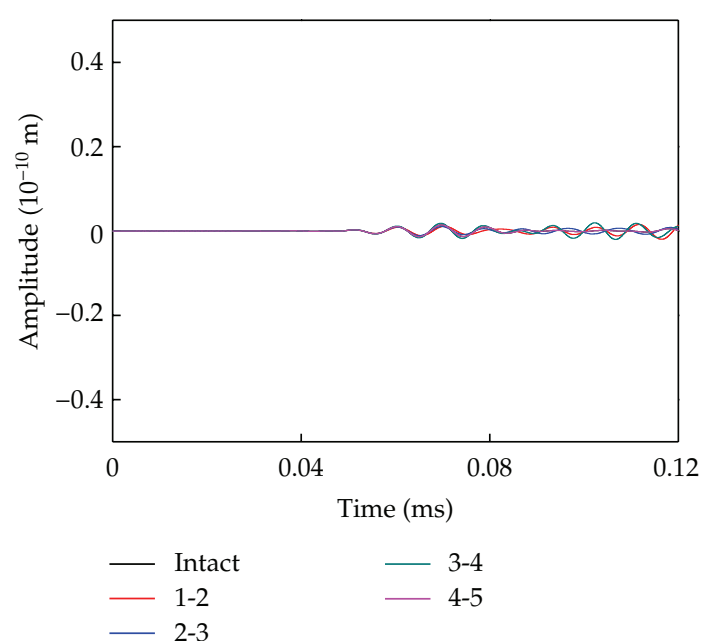

(b)

Figure 13: Displacement components in (a) the $x$-direction and (b) the $y$-direction at the point $\mathrm{B}$ as the delamination is located in different interfaces.

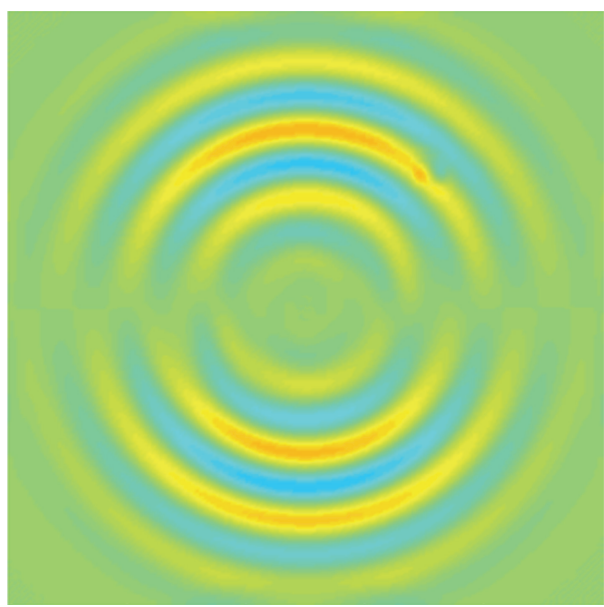

(a)

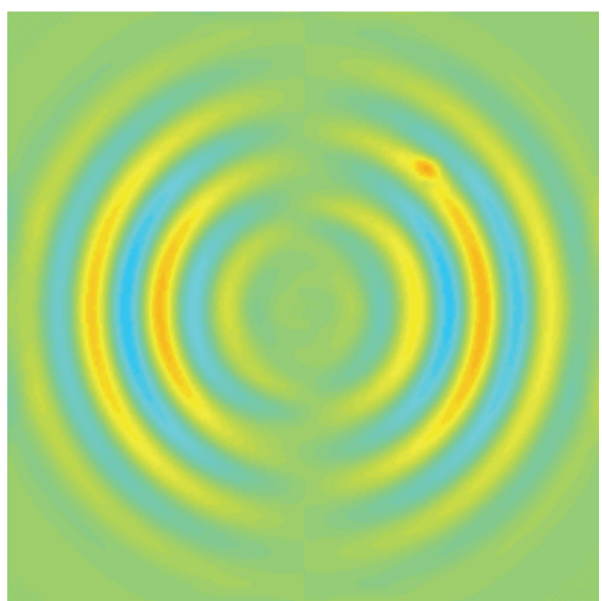

(b)

Figure 14: Displacement components in (a) the $x$-direction and (b) the $y$-direction of composite laminate at $0.069 \mathrm{~ms}$ with a delamination.

delamination located at all interfaces of the composite laminate. The displacement responses of the laminate in the $z$-direction at $0.173 \mathrm{~ms}$ are plotted in Figure 16.

Under the same excitation frequency, the scattered waves from delamination become clearer in the captured response at the point $B$ under the antisymmetricmode than that under symmetric mode. Hence, the antisymmetricmode can be used to detect smaller delamination than the symmetric mode, since the wavelength of the antisymmetricmode is less than that of the symmetric mode. In addition, according to the simulation results, the symmetric mode may be less sensitive to delamination located at certain interfaces. It can be expected that the antisymmetricmode is more suitable for identification of the delamination. 


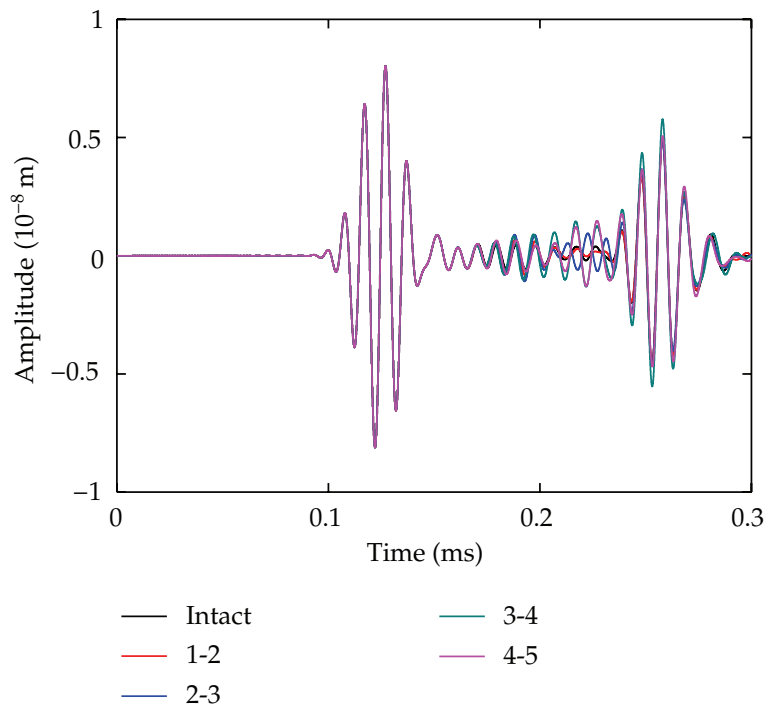

Figure 15: Displacement component in the $z$-direction at the point B as the delaminations are located in different interfaces.

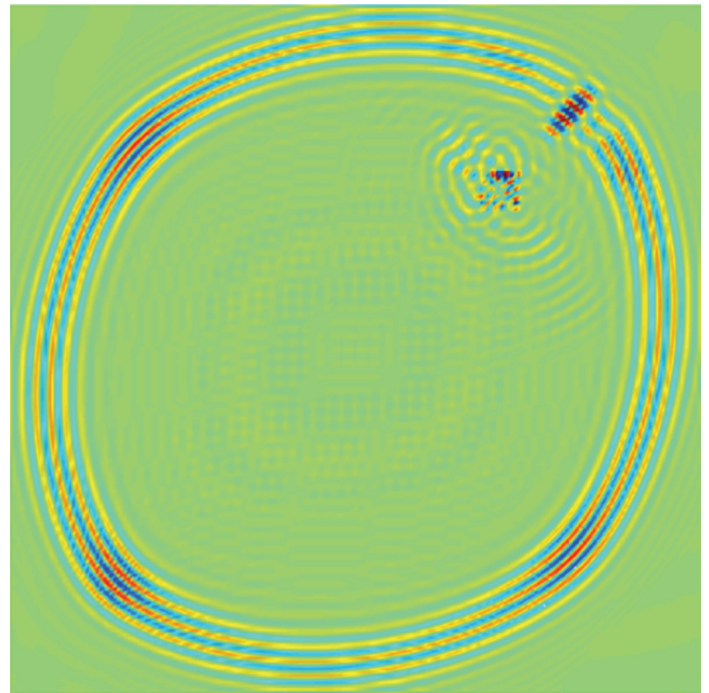

Figure 16: Displacement component in the $z$-direction of composite laminate at $0.173 \mathrm{~ms}$ with a delamination.

The scattered waves are very weak compared with the incident wave, resulting in that the reflected wave packet is difficult to identify delamination in such composite materials with a high attenuation ratio. Fortunately, the transmitted wave has been affected a lot, as shown in Figure 16. 


\section{Conclusions}

A three-dimensional spectral element method is developed to investigate the wave propagation characteristics in composite laminates in the present study. Three types of laminates, namely, unidirectional $[0]_{8}$, cross-ply $\left[0_{2} / 90_{2}\right]_{s}$, and quasi-isotropic $[-45 / 45 / 0 / 90]_{s}$ laminates are modeled using 3D spectral elements, and the laminates are excited using two forces in-phase and out-of-phase to generate the symmetric mode and antisymmetricmode respectively. It is demonstrated that the proposed 3D spectral element method can be efficiently and effectively used to simulate wave propagation in composite laminates. Complexity of wave propagation characteristic is also demonstrated even for a single Lamb wave mode in composite laminates. Finally, interactions between the Lamb wave mode and a delamination are analyzed. It is concluded that symmetric mode of Lamb wave may be insensitive to delamination in certain interfaces in the laminate. And, therefore, it is essential to understand wave propagation characteristics in composite laminate when Lamb-wave-based structural health monitoring strategy is carried out.

\section{Appendix}

The analytical wave front can be calculated as follows.

Consider wave propagation solutions in the following form:

$$
\{u, v, w\}=(1, V, W) U e^{\left[i \xi\left(x_{1}+\alpha x_{3}\right)-\omega t\right]},
$$

where $\xi$ is the wave number, $\omega$ is the circular frequency, $\alpha$ is still an unknown parameter, and $V$ and $W$ are ratios of the displacement amplitudes of $v$ and $w$, respectively. The choice of the solution leads to the three coupled equations that can be written as

$$
\left[\begin{array}{lll}
K_{11} & K_{12} & K_{13} \\
K_{21} & K_{22} & K_{23} \\
K_{31} & K_{32} & K_{33}
\end{array}\right]\left[\begin{array}{l}
U_{1} \\
U_{2} \\
U_{3}
\end{array}\right]=0,
$$

where the elements of the matrix $K$ are

$$
\begin{aligned}
& K_{11}=C_{11}+C_{55}-\rho c^{2}, \\
& K_{12}=C_{16}+C_{45} \alpha^{2}, \\
& K_{13}=\left(C_{13}+C_{55}\right) \alpha, \\
& K_{22}=C_{66}-\rho c^{2}+C_{44} \alpha^{2}, \\
& K_{23}=\left(C_{36}+C_{45}\right) \alpha, \\
& K_{33}=C_{55}-\rho c^{2}+C_{33} \alpha^{2} .
\end{aligned}
$$


The existence of nontrivial solutions for $U_{1}, U_{2}$, and $U_{3}$ demands the vanishing of the determinant of the matrix $K$ and yields the sixth-degree polynomial equation:

$$
\alpha^{6}+A_{1} \alpha^{4}+A_{2} \alpha^{2}+A_{3}=0
$$

There are six roots of this equation, which correspond to the three sets of mode pairs. For each $\alpha_{q}, q=1,2, \ldots, 6$, the displacement ratios $V_{q}=U_{2 q} / U_{1 q}$ and $W_{q}=U_{3 q} / U_{1 q}$ can be expressed as

$$
\begin{aligned}
V_{q} & =\frac{K_{11}\left(\alpha_{q}\right) K_{23}\left(\alpha_{q}\right)-K_{13}\left(\alpha_{q}\right) K_{12}\left(\alpha_{q}\right)}{K_{13}\left(\alpha_{q}\right) K_{22}\left(\alpha_{q}\right)-K_{12}\left(\alpha_{q}\right) K_{23}\left(\alpha_{q}\right)} \\
W_{q} & =\frac{K_{11}\left(\alpha_{q}\right) K_{23}\left(\alpha_{q}\right)-K_{12}\left(\alpha_{q}\right) K_{13}\left(\alpha_{q}\right)}{K_{12}\left(\alpha_{q}\right) K_{33}\left(\alpha_{q}\right)-K_{23}\left(\alpha_{q}\right) K_{13}\left(\alpha_{q}\right)} .
\end{aligned}
$$

The formal solutions for the displacements and stresses in the expanded matrix form

$$
\left[\begin{array}{l}
u_{1} \\
u_{2} \\
u_{3} \\
\sigma_{33} \\
\sigma_{13} \\
\sigma_{23}
\end{array}\right]=\left[\begin{array}{cccccc}
1 & 1 & 1 & 1 & 1 & 1 \\
V_{1} & V_{1} & V_{3} & V_{3} & V_{5} & V_{5} \\
W_{1} & -W_{1} & W_{3} & -W_{3} & W_{5} & -W_{5} \\
D_{11} & D_{11} & D_{13} & D_{13} & D_{15} & D_{15} \\
D_{21} & -D_{21} & D_{23} & -D_{23} & D_{25} & D_{25} \\
D_{31} & -D_{31} & D_{33} & -D_{33} & D_{35} & -D_{35}
\end{array}\right]\left[\begin{array}{l}
U_{11} E_{1} \\
U_{12} E_{2} \\
U_{13} E_{3} \\
U_{14} E_{4} \\
U_{15} E_{5} \\
U_{16} E_{6}
\end{array}\right],
$$

where

$$
\begin{gathered}
E_{q}=e^{i \xi \alpha_{q} x_{3}}, \\
D_{1 q}=i \xi\left(C_{13}+C_{36} V_{q}+C_{33} \alpha_{q} W_{q}\right), \\
D_{2 q}=i \xi\left[C_{55}\left(\alpha_{q}+W_{q}\right)+C_{45} \alpha_{q} V_{q}\right), \\
D_{3 q}=i \xi\left[C_{45}\left(\alpha_{q}+W_{q}\right)+C_{44} \alpha_{q} V_{q}\right), \\
q=1,2, \ldots, 6 .
\end{gathered}
$$

\section{Acknowledgments}

The authors are grateful for the support received from the National Natural Science Foundation of China (NSFC nos. 11072148 and 11061160491), Research Project of State Key Laboratory of Mechanical System and Vibration (MSV201110), and the National High Technology Research and Development Program of China (no. 2009AA044800). 


\section{References}

[1] L. Ye, Y. Lu, Z. Su, and G. Meng, "Functionalized composite structures for new generation airframes: a review," Composites Science and Technology, vol. 65, no. 9, pp. 1436-1446, 2005.

[2] Z. Su, L. Ye, and Y. Lu, "Guided Lamb waves for identification of damage in composite structures: a review," Journal of Sound and Vibration, vol. 295, no. 3-5, pp. 753-780, 2006.

[3] A. Raghavan and C. E. S. Cesnik, "Review of guided-wave structural health monitoring," Shock and Vibration Digest, vol. 39, no. 2, pp. 91-114, 2007.

[4] Z. Su and L. Ye, "An intelligent signal processing and pattern recognition technique for defect identification using an active sensor network," Smart Materials and Structures, vol. 13, no. 4, pp. 957969, 2004.

[5] Z. Su and L. Ye, "Lamb wave propagation-based damage identification for quasi-isotropic CF/EP composite laminates using artificial neural algorithm: part II-implementation and validation," Journal of Intelligent Material Systems and Structures, vol. 16, no. 2, pp. 113-125, 2005.

[6] X. Wang, G. Foliente, Z. Su, and L. Ye, "Multilevel decision fusion in a distributed active sensor network for structural damage detection," Structural Health Monitoring, vol. 5, no. 1, pp. 45-58, 2006.

[7] N. Hu, S. Takahito, F. Hisao, and Z. Su, “Damage identification of metallic structures using $\mathrm{A}_{0}$ mode of lamb waves," Structural Health Monitoring, vol. 7, no. 3, pp. 271-285, 2008.

[8] J. C. Strikwerda, Ed., Finite Difference Schemes and Partial Differential Equations, 2nd edition, 2004.

[9] J. C. Strikwerda, Finite Difference Schemes and Partial Differential Equations, Wadsworth Publishing Company, 1989.

[10] O. C. Zienkiewicz, The Finite Element Method, McGraw-Hill, London, UK, 4th edition, 1989.

[11] Y. Liu, N. Hu, C. Yan, X. Peng, and B. Yan, "Construction of a Mindlin pseudospectral plate element and evaluating efficiency of the element," Finite Elements in Analysis and Design, vol. 45, no. 8-9, pp. 538-546, 2009.

[12] N. Hu, H. H. Wang, B. Yan, H. Fukunaga, D. R. Mahapatra, and S. Gopalakrishnan, "The partition of unity finite element method for elastic wave propagation in Reissner-Mindlin plates," International Journal for Numerical Methods in Engineering, vol. 70, no. 12, pp. 1451-1479, 2007.

[13] Y. Cho and J. L. Rose, "A boundary element solution for a mode conversion study on the edge reflection of Lamb waves," Journal of the Acoustical Society of America, vol. 99, no. 4, pp. 2097-2109, 1996.

[14] X. G. Zhao and J. L. Rose, "Boundary element modeling for defect characterization potential in a wave guide," International Journal of Solids and Structures, vol. 40, no. 11, pp. 2645-2658, 2003.

[15] A. Bergamini and F. Biondini, "Finite strip modeling for optimal design of prestressed folded plate structures," Engineering Structures, vol. 26, no. 8, pp. 1043-1054, 2004.

[16] D. J. Dawe, "Use of the finite strip method in predicting the behaviour of composite laminated structures," Composite Structures, vol. 57, no. 1-4, pp. 11-36, 2002.

[17] P. P. Delsanto and M. Scalerandi, "A spring model for the simulation of the propagation of ultrasonic pulses through imperfect contact interfaces," Journal of the Acoustical Society of America, vol. 104, no. 5, pp. 2584-2591, 1998.

[18] P. P. Delsanto, R. S. Schechter, H. H. Chaskelis, R. B. Mignogna, and R. Kline, “Connection machine simulation of ultrasonic wave propagation in materials. II: the two-dimensional case," Wave Motion, vol. 20, no. 4, pp. 295-314, 1994.

[19] P. P. Delsanto, R. S. Schechter, and R. B. Mignogna, "Connection machine simulation of ultrasonic wave propagation in materials III: the three-dimensional case," Wave Motion, vol. 26, no. 4, pp. 329 339, 1997.

[20] P. P. Delsanto, T. Whitcombe, H. H. Chaskelis, and R. B. Mignogna, “Connection Machine simulation of ultrasonic wave propagation in materials. I. The one-dimensional case," Wave Motion, vol. 16, no. 1, pp. 65-80, 1992.

[21] Y. Sohn and S. Krishnaswamy, "Mass spring lattice modeling of the scanning laser source technique," Ultrasonics, vol. 39, no. 8, pp. 543-551, 2002.

[22] B. C. Lee and W. J. Staszewski, "Lamb wave propagation modelling for damage detection: II. Damage monitoring strategy," Smart Materials and Structures, vol. 16, no. 2, article 004, pp. 260-274, 2007.

[23] B. C. Lee and W. J. Staszewski, "Lamb wave propagation modelling for damage detection: I. twodimensional analysis," Smart Materials and Structures, vol. 16, no. 2, article 003, pp. 249-259, 2007.

[24] J. F. Doyle, Wave Propagation in Structures, Springer, New York, NY, USA, 1989.

[25] A. T. Patera, "A spectral element method for fluid dynamics: laminar flow in a channel expansion," Journal of Computational Physics, vol. 54, no. 3, pp. 468-488, 1984. 
[26] N. Hu, H. Fukunaga, M. Kameyama, D. R. Mahapatra, and S. Gopalakrishnan, "Analysis of wave propagation in beams with transverse and lateral cracks using a weakly formulated spectral method," Journal of Applied Mechanics, vol. 74, no. 1, pp. 119-127, 2007.

[27] M. Krawczuk, M. Palacz, and W. Ostachowicz, "The dynamic analysis of a cracked Timoshenko beam by the spectral element method," Journal of Sound and Vibration, vol. 264, no. 5, pp. 1139-1153, 2003.

[28] D. Komatitsch, C. Barnes, and J. Tromp, "Simulation of anisotropic wave propagation based upon a spectral element method," Geophysics, vol. 65, no. 4, pp. 1251-1260, 2000.

[29] D. Komatitsch and J. Tromp, "Introduction to the spectral element method for three-dimensional seismic wave propagation," Geophysical Journal International, vol. 139, no. 3, pp. 806-822, 1999.

[30] R. Sridhar, A. Chakraborty, and S. Gopalakrishnan, "Wave propagation analysis in anisotropic and inhomogeneous uncracked and cracked structures using pseudospectral finite element method," International Journal of Solids and Structures, vol. 43, no. 16, pp. 4997-5031, 2006.

[31] P. Kudela, M. Krawczuk, and W. Ostachowicz, “Wave propagation modelling in 1D structures using spectral finite elements," Journal of Sound and Vibration, vol. 300, no. 1-2, pp. 88-100, 2007.

[32] P. Kudela, A. Zak, M. Krawczuk, and W. Ostachowicz, "Modelling of wave propagation in composite plates using the time domain spectral element method," Journal of Sound and Vibration, vol. 302, no. 4-5, pp. 728-745, 2007.

[33] A. Zak, M. Krawczuk, and W. Ostachowicz, "Propagation of in-plane waves in an isotropic panel with a crack," Finite Elements in Analysis and Design, vol. 42, no. 11, pp. 929-941, 2006.

[34] H. Peng, G. Meng, and F. Li, "Modeling of wave propagation in plate structures using three-dimensional spectral element method for damage detection," Journal of Sound and Vibration, vol. 320, no. 4-5, pp. 942-954, 2009.

[35] N. Hu, T. Shimomukai, C. Yan, and H. Fukunaga, “Identification of delamination position in cross-ply laminated composite beams using S0 Lamb mode," Composites Science and Technology, vol. 68, no. 6, pp. 1548-1554, 2008.

[36] L. Wang and F. G. Yuan, "Group velocity and characteristic wave curves of Lamb waves in composites: modeling and experiments," Composites Science and Technology, vol. 67, no. 7-8, pp. 1370-1384, 2007.

[37] D. Komatitsch, S. Tsuboi, and J. Tromp, The Spectral-Element Method in Seismology, vol. 157 of Geophysical Monograph, American Geophysical Union, 2005.

[38] A. Lonkar and F. K. Chang, "Development of SEM-based PESEA code for modeling PZT induced acousto-ultrasonic waves propagation in metallic and composite structures," in Proceedings of the 8th International Workshop on Structural Health Monitoring, vol. 2, pp. 2512-2520, Stanford, Calif, USA, 2011.

[39] P. Kudela and W. Ostachowicz, "Spectral elements with electro-mechanical coupling for elastic wave modeling in 3D solids," in Proceedings of the 4th European Conference on Computational Mechanics, Solids, Structures and Coupled Problems in Engineering, Paris, France, May 2010. 


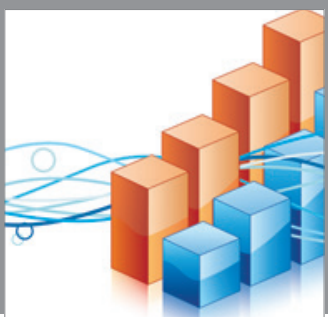

Advances in

Operations Research

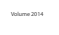

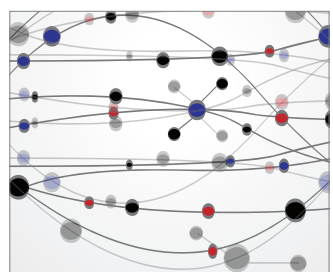

\section{The Scientific} World Journal
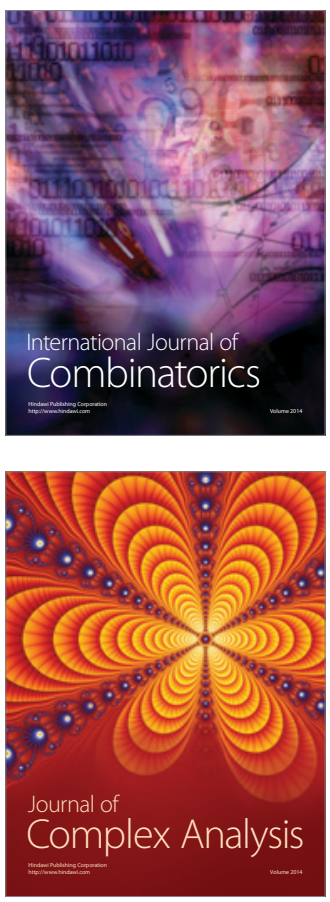

International Journal of

Mathematics and

Mathematical

Sciences
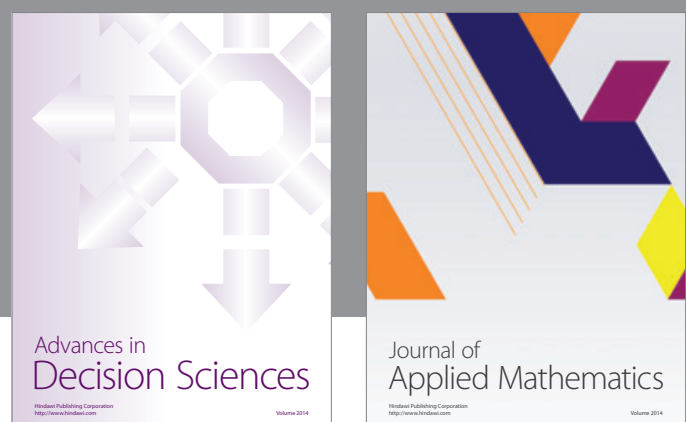

Journal of

Applied Mathematics
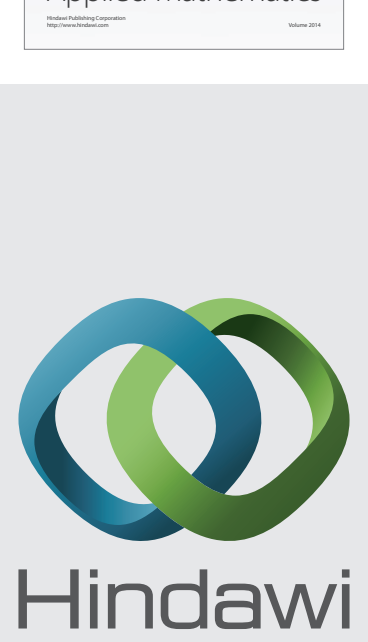

Submit your manuscripts at http://www.hindawi.com
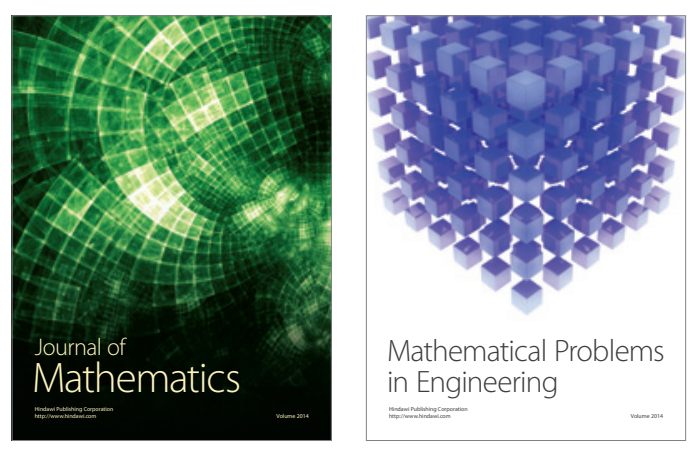

Mathematical Problems in Engineering
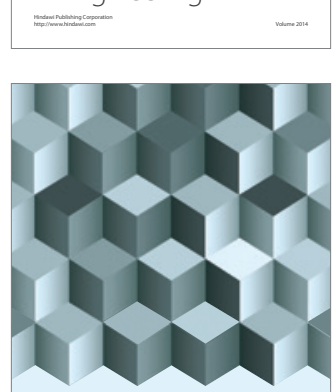

Journal of

Function Spaces
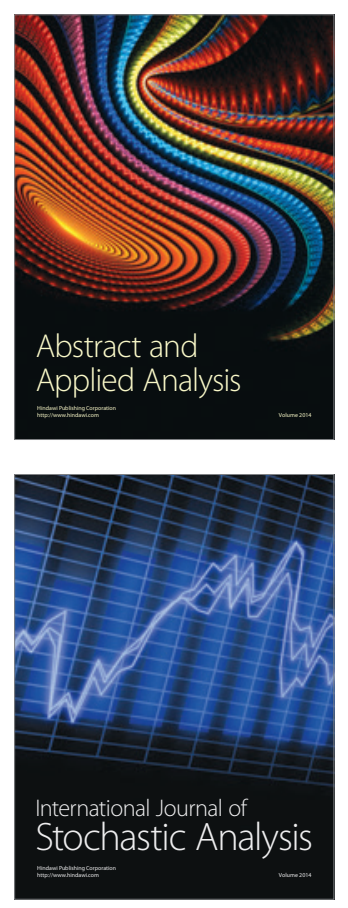

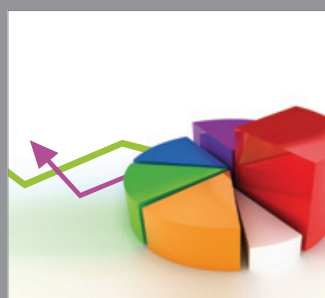

ournal of

Probability and Statistics

Promensencen
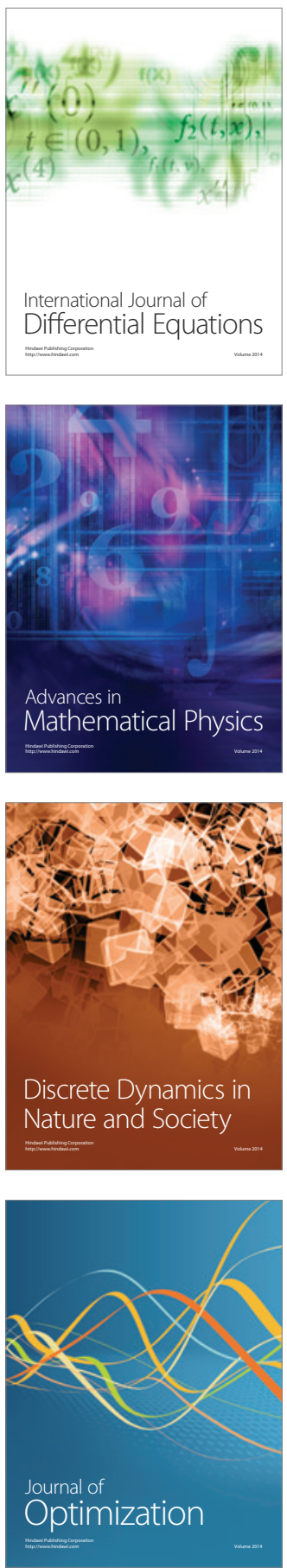